\title{
Clinical Characteristics of Ureteral Duplication in Children
}

\author{
Min Ji Park, M.D. ${ }^{1}$ \\ Hee Sun Baek, M.D.' \\ Hae Min Jang, M.D.' \\ Jun Nyung Lee, M.D. ${ }^{2}$ \\ Sung Kwang Chung, M.D. ${ }^{2}$ \\ Shin Young Jeong, M.D. ${ }^{3}$ \\ So Mi Lee, M.D. ${ }^{4}$ \\ Min Hyun Cho, M.D. ${ }^{1}$
}

Department of Pediatrics ${ }^{1}$, Kyungpook

National University, School of

Medicine, Department of Urology ${ }^{2}$,

Kyungpook National University,

School of Medicine, Department

of Nuclear Medicine ${ }^{3}$, Kyungpook

National University, School of

Medicine, Department of Radiology 4 ,

Kyungpook National University,

School of Medicine, Daegu, Republic

of Korea

Corresponding author:

Min Hyun Cho, M.D.

Department of Pediatrics, Kyungpook

National University Hospital, 130

Dongdeok-ro, Jung-gu, Daegu 41944,

Republic of Korea

Tel: +82-53-200-5719

Fax: $+82-53-425-6683$

E-mail:chomh@knu.ac.kr

Received: 30 August 2019

Revised: 4 October 2019

Accepted: 8 October 2019

This is an open-access article distributed under the terms of the Creative Commons Attribution Non-Commercial License (http:// creativecommons.org/licenses/by-nc/4.0/) which permits unrestricted non-commercial use, distribution, and reproduction in any medium, provided the original work is properly cited.

Copyright (C) 2019The Korean Society of Pediatric Nephrology
Purpose: Ureteral duplication is a relatively common congenital urinary tract abnormality that can be associated with various clinical problems such as vesicoureteral reflux (VUR), hydronephrosis, and ectopic ureters. The purpose of this study was to analyze the clinical characteristics of pediatric patients with recently diagnosed ureteral duplication and to identify any differences from those described in previous reports.

Methods: We retrospectively reviewed the clinical characteristics and course of pediatric patients who were diagnosed with ureteral duplication between January 2008 and June 2017.

Results: A total of 32 pediatric patients were diagnosed with ureteral duplication during the study period. The male to female ratio was 1:2.2. Twenty-seven patients (84.4\%) were first diagnosed with ureteral duplication at less than 3 months of age, and 26 (81.3\%) were first diagnosed by prenatal ultrasonography. Four of the 32 patients were diagnosed with bilateral ureteral duplication, for a total of 36 occurrences of ureteral duplication. In 17 occurrences of complete ureteral duplication (47.2\%), other urinary tract anomalies were also found; namely, ureterocele (7), VUR (11), and ectopic ureter (5). However, none of the patients with incomplete ureteral duplication had ureterocele orVUR.

Conclusions: With the advent of routine prenatal ultrasound, ureteral duplication is being diagnosed earlier than was previously possible, enabling timely treatment of the various accompanying urinary tract anomalies. Multicenter studies are needed to establish guidelines for standardized evaluation and treatment of ureteral duplication.

Key words: Ureter, Vesicoureteral reflux, Children

\section{Introduction}

Ureteral duplication is a congenital anomaly in which the ureteric bud, the embryonic origin of the ureter, divides or occurs twice and connects two ureters to one kidney ${ }^{1,2}$. This is one of the most common urogenital malformations occurring in approximately $1 \%$ of the population; it can be accompanied by various clinical complications, such as vesicoureteral reflux (VUR), hydronephrosis, and ectopic ureter ${ }^{1-3}$. Previously, ureteral duplications were diagnosed during hospital visit for urinary tract infection or fever. More recently, in a Korean study published in 2003, $47 \%$ of patients with ureteral duplication were diagnosed with prenatal ultrasonography, and 36\% were diagnosed during evaluation of fever or urinary tract infection, reflecting the 
changing process of ureteral duplication diagnosis ${ }^{4)}$.

The purpose of this study was to analyze the clinical characteristics of pediatric patients recently diagnosed with ureteral duplication and to compare the characteristics with those described in previous reports.

\section{Material and methods}

We retrospectively reviewed the medical records of 32 pediatric patients who were diagnosed with ureteral duplication at Kyungpook National University Hospital between January 2008 and June 2017. The initial diagnosis of ureteral duplication was usually made by ultrasonography, performed during the prenatal period in most cases. Intravenous pyelogram, the previous main diagnostic tool for ureteral duplication, was seldom performed due to the radiation risk and lack of additional benefit compared with ultrasonography. With ultrasonography, ureteral dilation, presence of ureterocele in the bladder, and severity of hydronephrosis were evaluated simultaneously. VUR was diagnosed only by voiding cystourethrogram. To distinguish between complete and incomplete types of ureteral duplication, additional evaluations including diuretic renogram and magnetic resonance urography were performed; diagnostic cystoscopy was performed to identify the orifice of the ectopic ureter in some patients. Additional data included the patients' age, sex, clinical manifestations at initial diagnosis, type of duplex system, and other urological anomalies.

The Student's t-test was used to analyze continuous variables. A $P$ value $<0.05$ was considered statistically significant. All statistical analyses were performed with the R software, version 3.4.3 (R Foundation for Statistical Computing, Vienna, Austria).

\section{Results}

During the study period, 32 infants and children were diagnosed with ureteral duplication at our hospital. Of them, 22 were female (68.7\%) and 10 were male (31.3\%). In 27 patients $(84.4 \%)$, ureteral duplication was diagnosed before the age of 3 months and in 31 (96.9\%), ureteral dupli- cation was diagnosed during the first 12 months. In other words, most of these patients were first diagnosed with ureteral duplication by age three months, and almost all were diagnosed by age 12 months (Table 1).

Ureteral duplication was first detected by prenatal ultrasonography in $81.3 \%$ of the patients $(n=26)$; in $15.6 \%(n=5)$, ureteral duplication was detected during a visit to the hospital due to fever and urinary tract infection. No patients presented with urinary incontinence due to ectopic ureter as an initial chief concern.

Because 4 of the 32 included patients (12.5\%) were had bilateral ureteral duplication, a total of 36 occurrences of ureteral duplication were investigated. Among these, 17 occurrences (47.2\%) were compatible with the complete type of ureteral duplication and 19 cases (52.8\%) with the incomplete type. There was no significant difference between the right kidney to left kidney ratios: 9 to 8 for the complete type and 11 to 8 for the incomplete type (Table 1).

Other urinary tract anomalies associated with the complete type of ureteral duplication were: ureterocele in 7 cases $(19.4 \%$ of total 36 cases/41\% of 17 cases with the complete type), vesicoureteral reflux in 11 cases (30.6\% of total 36 cases $/ 65 \%$ of 17 cases with the complete type), and

Table 1. Clinical Characteristics of Patients with Ureteral Duplication

\begin{tabular}{lc}
\hline Patients $(\mathrm{n}=32) /$ Cases of Ureter duplication $(\mathrm{n}=36)$ & \\
\hline Sex & $10(31.3 \%)$ \\
Male & $22(68.7 \%)$ \\
Female & \\
Age at diagnosis (Mo) & $27(84.4 \%)$ \\
$<3$ Mo & $4(12.5 \%)$ \\
$3-12$ Mo & $1(3.1 \%)$ \\
$>12$ Mo & $26(81.3 \%)$ \\
Clinical Manifestations at Initial Diagnosis & $5(15.6 \%)$ \\
Prenatal USG & $1(3.1 \%)$ \\
Fever \& UTI & \\
others & $17(47.2 \%)(\mathrm{R}: \mathrm{L}=9: 8)$ \\
Type of duplication ( $\mathrm{n}=36)$ & $19(52.8 \%)(\mathrm{R}: \mathrm{L}=11: 8)$ \\
Complete & \\
Incomplete & $7(19.4 \%)$ \\
Combined urinary tract anomalies $(\mathrm{n}=36)$ & $11(30.6 \%)$ \\
Ureterocele & $5(13.9 \%)$ \\
\hline VUR & \\
Ectopic ureter & \\
\hline
\end{tabular}

Abbreviations: Mo, months; USG, ultrasonography; UTI, urinary tract infection; $R$, right kidney; L, left kidney; VUR, vesicoureteral reflux. 
ectopic ureter in 5 cases (13.9\% of total 36 cases/29\% of 17 cases with the complete type). In contrast, among 19 cases of incomplete ureteral duplication, none was associated with ureterocele or VUR (Table 2).

VUR was confirmed in the upper moiety of the ureteral duplication ( 2 cases), in the lower moiety (4 cases), in both the upper and lower moieties (2 cases), and in the upper and lower moieties of the ureteral duplication and the contralateral normal kidney ( 3 cases) (Table 3 and Fig. 1). Three cases of VUR were treated surgically (e.g., with ureteroneocystostomy), 4 cases were managed by observation and did not develop urinary tract infection or other complications, and in 4 cases, VUR resolved spontaneously.

In the 5 cases of ectopic ureter associated with ureteral duplication, the ectopic ureter was located in the bladder neck (2), urethra (2), and vagina (1) (Table 3). In all 7 cases with ureterocele, transurethral resection was performed, followed by ureteroureterostomy in 6 cases as the definitive operative treatment. Notably, one patient with ureterocele who underwent transurethral resection developed new VUR postoperatively.

Table 2. Other Urological Anomalies in Relation to Ureteral Duplication

\begin{tabular}{lcrc}
\hline Case $(n=36)$ & Presence & $\begin{array}{c}\text { Complete type } \\
(n=17)\end{array}$ & $\begin{array}{c}\text { Incomplete type } \\
(n=19)\end{array}$ \\
\hline VUR & + & $11(65 \%)$ & 0 \\
Ureterocele & - & $6(35 \%)$ & 19 \\
& + & $7(41 \%)$ & 0 \\
Ectopic ureter & - & $10(59 \%)$ & 19 \\
& + & $5(29 \%)$ & 0 \\
& - & $12(71 \%)$ & 19
\end{tabular}

Abbreviation: VUR, vesicoureteral reflux.

\begin{tabular}{|c|c|}
\hline \multicolumn{2}{|c|}{ Complete type of ureteral duplication $(n=17)$} \\
\hline \multirow[t]{4}{*}{$\operatorname{VUR}(n=11)$} & Upper moiety: 2 \\
\hline & Lower moiety: 4 \\
\hline & Upper and lower moiety: 2 \\
\hline & $\begin{array}{l}\text { Upper, lower moiety and contralateral } \\
\text { normal kidney: } 3\end{array}$ \\
\hline \multirow[t]{3}{*}{ Orifice of ectopic ureter $(n=5)$} & Bladder neck: 2 \\
\hline & Urethra: 2 \\
\hline & Vagina: 1 \\
\hline
\end{tabular}

Abbreviation: VUR, vesicoureteral reflux.

\section{Discussion}

Ureteral duplication is the most common congenital anomaly of the urinary tract, with an estimated incidence in the general population of $0.8-5 \%$ from autopsy studies ${ }^{1,}$ ${ }^{2,5)}$. The congenital malformation consists of two ureters connected to one kidney, resulting from duplication or division of the ureteric bud, the embryonic origin of the ureters $^{1,3)}$. The superior bud derives from the upper pole of a duplicated kidney and the inferior bud derives from the lower pole. Generally, superior bud is carried inferiorly along with any derivative of the distal mesonephric duct, so the ectopic ureter drains the upper pole of the duplicated kidney and the two ureters cross each other ${ }^{6}$. Therefore, the orifice of the ureter communicating with the upper pole in the complete type of ureteral duplication is located more medial and inferior than the normal position, but that of the ureter communicating with the lower pole is lo-

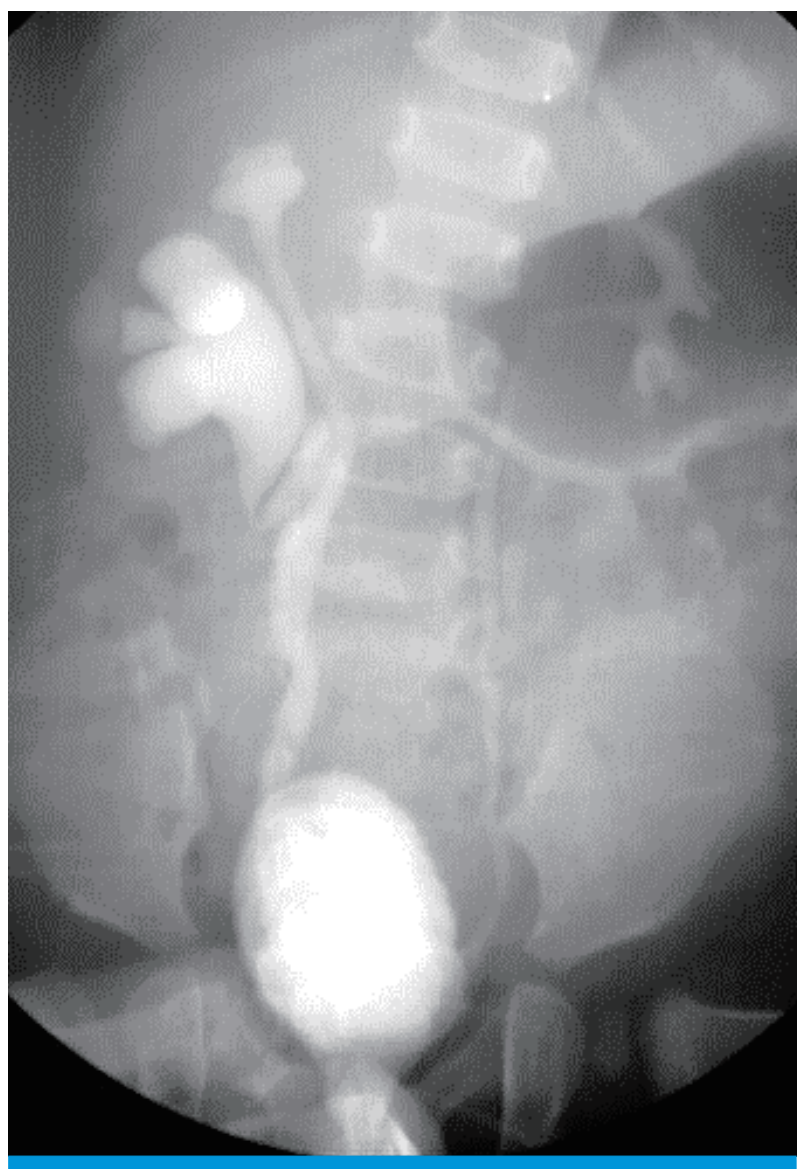

Fig. 1. This voiding cystourethrogram shows vesicoureteral reflux into the upper \& lower moiety of ureteral duplication in right kidney and the contralateral normal left kidney. 
cated more lateral and superior than the normal position, as described by the Weigert-Meyer rule ${ }^{6-8)}$. As a result, dysplastic change and hydronephrosis due to ureteral obstruction more usually occur in the upper pole of a duplicated kidney, and VUR more usually occurs at the lower pole ${ }^{6,9)}$. Incomplete type of ureteral duplication is thought to arise from premature bifurcation of the ureteric bud, before entering the metanephric blastema and not clinically significant in most cases ${ }^{10)}$.

In the past, ureteral duplication was diagnosed during hospital visits for urinary tract infection or fever, but more recently, the diagnosis was often made before the development of complication. In an article published in $2003^{4}$, $47 \%$ of cases of ureteral duplication were diagnosed by prenatal ultrasonography and 36 percent were diagnosed in patients with fever or urinary tract infection. In our study, $81.3 \%$ of cases were first detected by prenatal ultrasonography, and $15.6 \%$ were diagnosed during a visit to the hospital due to fever and urinary tract infection. Also, no patient visited the hospital with urinary incontinence due to an ectopic ureter as an initial chief concern. Our results show that ureteral duplication is being diagnosed earlier, by routine fetal ultrasound, than described in previous reports.

It has been reported that ureteral duplication generally occurs more commonly in female patients than in male patients, and this anomaly is bilateral in $17-33 \%$ of cases. Additionally, a total of $60 \%$ of duplex systems have incomplete ureters duplication, with $40 \%$ showing complete ureteral duplication1). Similarly, in our study, female patients (68.7\%) outnumbered male patients (31.3\%), and incomplete types (52.8\%) were more common than complete types (47.2\%).

Most patients with incomplete types of ureteral duplication are clinically asymptomatic and the anomaly may be undetected. However, the complete type of ureteral duplication may be associated with an ectopic ureter, VUR, or ureterocele, and with symptoms such as obstruction, urinary incontinence, or urinary tract infection ${ }^{11,12)}$. Accordingly, in our study, cases of complete ureteral duplication had accompanying by VUR, ureterocele, and ectopic ureter; in contrast, none of the incomplete type cases we evaluated had associated ureterocele or VUR.

VUR is more often found in association with ureteral duplication than in a single system and appears in $70 \%$ of patients with infection and ureteral duplication ${ }^{13)}$. Lowgrade VUR can be managed with antibiotics and careful observation, but high-grade VUR requires more intensive management. If kidney damage increases or antibiotic prevention fails, surgery must be performed ${ }^{14)}$. In our study, 4 patients with high-grade VUR were treated surgically. 8 patients with low-grade VUR who were managed by observation, presented no urinary tract infection or spontaneous resolution of VUR.

Another malformation associated with ureteral duplication, ectopic ureter, occurs 2 to 3 times more commonly in female patients than in male patients ${ }^{15}$. When the urethra is located in the distal region of the sphincter, female patients may have urinary incontinence ${ }^{15)}$. In this study, 5 cases of ectopic ureter were identified, of which 3 occurred in girls and 2 in boys.

Obstruction of the ureteral duplication most often occurs due to the presence of ureterocele ${ }^{16)}$. This complication usually occurs 8 times more often in female patients than in male patients ${ }^{17)}$. In our study, 7 cases of ureterocele were identified, including 6 cases in girls and 1 case in a boy. Initial and subsequent management of complete ureteral duplication with VUR or ureterocele has been controversial, and the management options have included partial nephrectomy, endoscopic decompression, and ureteroureterostomy ${ }^{11,18,19)}$. The first-line procedure for ureterocele in neonatal patients is endoscopic puncture; accordingly, in all 7 cases of ureterocele in this study, a transurethral resection was performed and in 6 of these cases, ureteroureterostomy was performed as the definitive treatment for complete ureteral duplication.

In conclusion, this study shows that ureteral duplication is being detected earlier through the routine performance of prenatal ultrasonography and that conservative management is more common than surgical management. However, additional evaluation and treatment protocols are needed to overcome the limitations of ultrasonography, such as difficulties in identifying the type of duplication, presence of VUR, and location of the orifice of ectopic ureter. 


\section{Conflict of interest}

The authors report no conflicts of interest. The authors alone are responsible for the content of the manuscript.

\section{Patient consent}

This study was approved by the institutional review board (IRB), and the consent was waived due to the nature of the retrospective study [IRB number 2019-09-020].

\section{References}

1. Whitten SM, Wilcox DT. Duplex systems. Prenat Diagn 2001;21: 952-7.

2. Thomas JC. Vesicoureteral reflux and duplex systems. Adv Urol 2008;651891.

3. Decter RM. Renal duplication and fusion anomalies. Pediatr Clin North Am 1997;44:1323-41.

4. Han JW, Hwang DH, Park JM, Lee JS, Han SW. Clinical manifestations of ureteral duplication in children. J Korean Soc Pediatr Nephrol 2003;7:189-96.

5. Monie IW. Double ureter in two human embryos. Anat Rec 1949; 103:195-204

6. Reisner DC, Elgethun MT, Heller MT, Klepchick PR, Hartman MS. Congenital and acquired disorders of ureteral course. Curr Probl Diagn Radiol 2017;46:151-60.

7. Dinanath P, Ashwini A, Annarao G, Nagaraj SJ. Bilateral complete duplex renal pelvis and ureters-a case report. Int J Anat Var 2011;
4:192-4.

8. Johnston JH. Urinary tract duplication in childhood. Arch Dis Child 1961;36:180-9

9. Dewan PA, Penington E, Jeyaseelan D. Upper pole pelviureteric junction obstruction. Pediatr Surg Int 1998;13:290-2.

10. Mathiot A, Bargy F, Beaudoin S, Barbet P. Uncommon ureteric ectopias: embryological implications. Anat Embryol (Berl) 2004; 207:489-93.

11. Rodrigues I, Estevão-Costa J, Fragoso AC. Complete Ureteral Duplication: Outcome of Different Surgical Approaches. Acta Med Port 2016;29:275-8.

12. Siomou E, Papadopoulou F, Kollios KD, Photopoulos A, Evagelidou E, Androulakakis P, et al. Duplex collecting system diagnosed during the first 6 years of life after a first urinary tract infection: a study of 63 children. J Urol 2006;175:678-82.

13. Privett J, Jeans W, Roylance J. The incidence and importance of renal duplication. Clin Radiol 1976;27:521-30.

14. Afshar K, Papanikolaou F, Malek R, Bagli D, Pippi-Salle JL, Khoury A. Vesicoureteral reflux and complete ureteral duplication. Conservative or surgical management? J Urol 2005;173:1725-7.

15. Rodriguez MM. Congenital anomalies of the kidney and the urinary tract (CAKUT). Fetal Pediatr Pathol 2014;33:293-320.

16. Jee LD, Rickwood AM, Williams MP, Anderson PA. Experience with duplex system anomalies detected by prenatal ultrasonography. J Urol 1993;149:808-10.

17. Fernbach SK, Feinstein KA, Spencer K, Lindstrom CA. Ureteral duplication and its complications. Radiographics 1997;17:109-27.

18. Blyth B, Snyder HM, Duckett JW. Antenatal diagnosis and subsequent management of hydronephrosis. J Urol 1993;149:693-8.

19. Husmann D, Strand B, Ewalt D, Clement M, Kramer S, Allen T. Management of ectopic ureterocele associated with renal duplication: a comparison of partial nephrectomy and endoscopic decompression. J Urol 1999;162:1406-9. 